\title{
Zur „Methode der Atome in Molekülen“
}

\author{
Von H. Preuss \\ Aus dem Max-Planck-Institut für Physik, Göttingen \\ (Z. Naturforschg. 12 a, 599—603 [1957] ; eingegangen am 29. Mai 1957)
}

\begin{abstract}
Im Rahmen der „Methode der Atome in Molekülen“ wird eine Vereinigung der Verfahren nach Moffitt und Bingel in der Weise vorgenommen, daß die Konvergenzbedingungen in beiden Verfahren erhalten bleiben. Das ergibt eine bessere Angleichung an die wirklichen Energiekurven.

Am Beispiel des $\mathrm{H}_{2}{ }^{+}$-Grundzustandes wird gezeigt, daß mit einer sehr groben Näherungsfunktion eine Bindungsenergie von etwa $-1,95 \mathrm{eV}$ bei einem Kernabstand $R$ von 2,3 at. E. resultiert. Das Moffirtsche Verfahren liefert in dieser Form der Näherung $-1,75 \mathrm{eV}$ und $R=2,6$ at. E., während in der BingeLschen Darstellung noch keine Bindung auftritt.

Beim $\mathrm{H}_{2}$-Molekül erhält man (zwei Konfigurationen) $-4,71 \mathrm{eV}$ bei einem Kernabstand von 1,466 at. E. Die entsprechenden Werte nach Mofritr sind $-4,35 \mathrm{eV}$ und 1,40 at. E., wenn vier Konfigurationen verwendet werden. Die wirklichen Werte betragen $-4,72 \mathrm{eV}$ und 1,40 at. E.
\end{abstract}

Die Rechenmethode zur quantenmechanischen Behandlung von Molekülen, die unter der Bezeichnung „Methode der Atome in Molekülen“ („method of atoms in molecules“) bekannt geworden ist ${ }^{1}$, geht von der Tatsache aus, daß Berechnungen nach den beiden konventionellen Näherungsverfahren, der Methode der Valenzstrukturen (HLSP-Methode) und der Methode der Molekülzustände (Mo-Methode), trotz größerem Rechenaufwand bezüglich ihrer Übereinstimmung mit experimentellen Daten oft unbefriedigend sind. Besonders dann, wenn es gilt, Anregungsenergien von Molekülen oder Trennungsenergien von einem Atom oder von Atomgruppen zu bestimmen. Der Grund liegt hauptsächlich darin, daß die beiden Verfahren die Energie eines Elektronenzustandes bezüglich eines Nullniveaus berechnen, bei dem entweder das Atom vollständig ionisiert vorliegt oder beim Molekül alle Atomkerne unendlich weit entfernt sind. Obwohl diese absoluten Energien zuweilen prozentual ziemlich genau berechnet werden können, sind die besagten Energiedifferenzen, die immer klein gegen diese Energien sind, mit größeren Fehlern behaftet.

Die „Methode der Atome in Molekülen“ versucht diese Mängel dadurch zu vermeiden, daß von bestimmten Grenzzuständen des vorliegenden Systems ausgegangen wird, deren Energien aus spektroskopischen Daten entnommen werden.

Wir kennen z. Zt. zwei solche Verfahrenswege. Einmal das Moffitrsche Verfahren ${ }^{1}$, welches von den Energien der unendlich weit entfernten Atome des Moleküls ausgeht, zum anderen hat kürzlich BINGeL $^{2}$ auf die Möglichkeit hingewiesen, als Ausgangspunkt das „vereinigte Atom“ anzusetzen, dessen Kernladung gleich der Summe der Kernladun- gen der im Molekül enthaltenen Atome ist. Die Energien der getrennten Atome bzw. die Energien des vereinigten Atoms sind als Nullniveau gedacht und werden aus den Ergebnissen der Atomspektroskopie entnommen. Im ersteren Falle werden nur die bei der Molekülbindung freiwerdenden Energien theoretisch berechnet, im zweiten Falle die relativen Molekülenergien auf die Zustände des vereinigten Atoms bezogen und nur diese behandelt.

Man wird also sicher sein, daß man nach diesen Verfahren für kleine oder für große Kernabstände eine bessere Berechnung der Elektronenterme wird durchführen können. Allerdings zeigt sich, daß das Moffitrsche Verfahren für kleine Kernabstände und die Methode nach Bingel für große Abstände der Atome zu teilweise schlechten Ergebnissen führt und keine sinnvolle Konvergenz für $R \rightarrow 0$ bzw. $R \rightarrow \infty$ liefert. Eine Verbesserung kann erreicht werden, indem man beide Methoden vereinigt, und zwar so, daß sich für $R \rightarrow \infty$ und $R \rightarrow 0$ die beiden Grenzfälle ergeben.

Für diese sind dann als Nullniveaus die getrennten Atome oder das vereinigte Atom anzunehmen, deren Energien spektroskopisch vorliegen sollen. In diesem Falle ist der Einfluß der bei Berechnungen von vielen Elektronen notwendig auftretenden Fehler am geringsten.

Ehe wir diesen Weg einschlagen, sollen noch einmal kurz die beiden Verfahren, soweit sie für das folgende notwendig sind, angegeben werden. Bezüglich einer ausführlicheren Diskussion sei auf die Zusammenfassung von BINGEL ${ }^{2}$ hingewiesen.

Der Hamilton-Operator eines Moleküls mit $N$ Atomen, deren Kernladungszahlen $Z_{\lambda}(\lambda=1,2, \ldots, N)$ sind, schreibt sich in atomaren Einheiten wie folgt: 


$$
\mathcal{H}=-\frac{1}{2} \sum_{i=1}^{n} \Delta_{i}-\sum_{n=1}^{n} \sum_{i=1}^{N} \frac{Z_{\lambda}}{r_{y i}}+\sum_{\substack{i=1 \\ i>k}}^{n} \sum_{k=1}^{n} \frac{1}{r_{i k}},
$$

wenn $n$ Elektronen vorliegen und die CoulombAbstoßung der Kerne weggelassen wird. (Diese kann bei festgehaltenen Kernkoordinaten als Konstante zu jedem Molekülterm zugeschlagen werden.)

Die beiden Verfahren unterscheiden sich durch die Zerlegung von $\mathcal{H}$. Nämlich einmal in die Form $\mathcal{H}=H_{\infty}+V_{\infty} \quad$ (Moffitrsches Verfahren)

$$
\text { mit } \begin{aligned}
H_{\infty} & =\sum_{i=1}^{N} H_{\lambda}, \\
V_{\infty} & =\sum_{i} \sum_{i} \frac{Z_{\lambda}}{r_{\lambda i}}+\sum_{i} \sum_{k} \frac{1}{r_{i k}},
\end{aligned}
$$

wobei $H_{\gamma}$ die Hamilton-Operatoren der einzelnen Atome $\lambda$ darstellen, und $V_{\infty}$ der Wechselwirkungsoperator der Elektronen aus verschiedenen Atomen oder zwischen Elektronen und fremden Kernen ist. Die Striche an den Summenzeichen weisen auf diese Einschränkung hin. Im anderen Falle setzt man

$$
\mathcal{H}=H_{0}+V_{0} \quad \text { (Bingelsches Verfahren) }
$$

mit

$$
\begin{aligned}
& H_{0}=-\frac{1}{2} \sum_{i=1}^{n} \Delta_{i}-\sum_{i=1}^{n} \frac{Z}{r_{i x}}+\sum_{\substack{i=1 \\
i>k}}^{n} \sum_{\substack{k=1 \\
i}}^{n} \frac{1}{r_{i k}}, \\
& V_{0}=\sum_{i=1}^{n}\left(\frac{Z}{r_{i x}}-\sum_{i=1}^{N} \frac{Z i}{r_{\lambda i}}\right) \text { und } \sum_{i=1}^{N} Z_{i}=Z,
\end{aligned}
$$

wobei jetzt $H_{0}$ der Operator des vereinigten Atoms bedeutet, welches an der Stelle $x$ liegt, dem Schwerpunkt der Kernladungen. Entsprechend der Zerlegungen (2) und (3) werden als Ausgangsfunktionen die strengen Eigenfunktionen $\varphi_{\lambda}$ der Atome $\lambda$ (4a) und des vereinigten Atoms genommen $\Phi(4 \mathrm{~b})$,

$$
\begin{aligned}
& H_{\lambda} \varphi_{\lambda r}=W_{\lambda r} \varphi_{\lambda r}, \\
& H_{0} \Phi_{r}=W_{r} \Phi_{r} ;
\end{aligned}
$$

$r$ steht für die Gesamtheit der Quantenzahlen.

Der nächste Schritt besteht darin, daß die Moleküleigenfunktionen $\Omega_{a}$ entweder nach dem vollständigen Funktionensystem der $\Phi_{r}$ oder $\psi_{s}$ entwickelî werden, wobei $\psi_{s}$ aus dem antisymmetrischen Produkt aller $\varphi_{\lambda r}$ entstanden ist und in $s$ die verschiedenen Konfigurationen darstellen soll, die sich aus den $r$ der Atome $\lambda$ zusammensetzen (7) :

$$
\begin{aligned}
\Omega_{\alpha} & =\sum_{s} \Gamma_{s a} \psi_{s}, \\
\Omega & =\sum_{r} \Gamma_{r a} \Phi_{r} .
\end{aligned}
$$

Es gilt also

mit

$$
H_{\infty} \psi_{s}=W_{s}^{\infty} \psi_{s}
$$

$$
W_{s}{ }^{\infty}=\sum_{\lambda=1}^{N} W_{\lambda, s}, \quad s=s(r, \lambda) .
$$

Setzt man (5 a) und (5 b) in (2) und (3) ein, so erhält man für jedes Verfahren eine Säkulargleichung für die Molekülenergien $E$,

$$
\begin{aligned}
\left|\mathfrak{S}_{\infty}-\mathfrak{M} E\right| & =0, \\
\left|\mathfrak{S}_{0}-\mathfrak{E} E\right| & =0
\end{aligned}
$$

mit den Matrizen $\mathfrak{S}_{\infty}, \mathfrak{F}_{0}$ und $\mathfrak{M}$, sowie der Einheitsmatrix $\mathcal{F}$, deren Elemente die folgenden Integrale sind:

$$
\begin{aligned}
{\left[\mathfrak{S}_{\infty}\right]_{i k} } & =\left(\psi_{i}^{*} \mathcal{H} \psi_{k}\right), \quad[\mathfrak{M}]_{i k}=\left(\psi_{i}^{*} \psi_{k}\right), \\
{\left[\mathfrak{S}_{0}\right]_{i k} } & =\left(\Phi_{i}^{*} \mathcal{H} \Phi_{k}\right), \quad \delta_{i k}=\left(\Phi_{i}^{*} \Phi_{k}\right) ;
\end{aligned}
$$

sie lassen sich zerlegen nach

$$
\begin{gathered}
\mathfrak{S}_{\infty}=\mathfrak{W}_{\infty} \mathfrak{M}+\mathfrak{V}_{\infty}, \\
\mathfrak{H}_{0}=\mathfrak{W}_{0}+\mathfrak{V}_{0}
\end{gathered}
$$

mit

$$
\begin{aligned}
{\left[\mathfrak{V}_{\infty}\right]_{i k} } & =\left(\psi_{i}^{*} V_{\infty} \psi_{k}\right), \quad\left[\mathfrak{W}_{\infty}\right]_{i k}=W_{i}^{\infty} \delta_{i k}, \\
{\left[\mathfrak{V}_{0}\right]_{i k} } & =\left(\Phi_{i}^{*} V_{0} \Phi_{k}\right), \quad\left[\mathfrak{W}_{0}\right]_{i k}=W_{i}^{0} \delta_{i k} .
\end{aligned}
$$

Für $R \rightarrow \infty$ gilt

$$
\mathfrak{S}_{\infty} \rightarrow \mathfrak{W}_{\infty} \quad \mathfrak{M} \rightarrow \mathfrak{F} \text { und } \mathfrak{B}_{\infty} \rightarrow 0,
$$

während sich für $R \rightarrow 0$ in Übereinstimmung mit den Annahmen

$$
\mathfrak{S}_{0} \rightarrow \mathfrak{W}_{0}, \quad \mathfrak{V}_{0} \rightarrow 0
$$

ergibt. Das ist die oben besprochene Zerlegung der Molekülenergie in die Energien der Grenzzustände. $\mathfrak{W}_{0}$ und $\mathfrak{W}_{\infty}$ werden aus den Spektren entnommen.

Damit $\mathscr{S}_{\infty}$ hermitesch ist, wird diese ersetzt durch

$$
\mathfrak{S}_{\infty}=\frac{1}{2}\left(\mathfrak{W}_{\infty} \mathfrak{M}+\mathfrak{M} \mathfrak{W} \mathfrak{W}_{\infty}\right)+\frac{1}{2}\left(\mathfrak{V}_{\infty}+\mathfrak{V}_{\infty}^{+}\right),
$$

wo $\mathfrak{V}_{\infty}^{+}$die zu $\mathfrak{B}_{\infty}$ konjugierte Matrix ist.

Infolge der Unkenntnis der exakten Funktionen $\Phi_{r}$ und $\psi_{s}$ können nur Näherungsfunktionen $\tilde{\Phi}_{r}$ und $\tilde{\psi}_{s}$ verwendet werden. Mit diesen werden dann Näherungsfunktionen für $\mathfrak{S}_{\infty}$ und $\tilde{S}_{0}$ gebildet, 
$\mathfrak{S}_{\infty} \approx \tilde{\mathfrak{W}} \infty=\frac{1}{2}\left[\mathfrak{W}_{\infty} \tilde{\mathfrak{M}}+\tilde{\mathfrak{M}} \mathfrak{W}_{\infty}\right]+\frac{1}{2}\left[\tilde{\mathfrak{V}}_{\infty}+\tilde{\mathfrak{V}}_{\infty}^{+}\right]$,

$\tilde{\mathfrak{S}}_{0} \approx \tilde{\mathfrak{S}}_{0}=\mathfrak{W}_{0}+\tilde{\mathfrak{V}}_{0}$,

mit denen im Säkularproblem gerechnet wird. $\tilde{5}_{\infty}$ und $\tilde{\mathfrak{S}}_{0}$ zeigen in der Form (14) mit den genauen Matrizen $\mathfrak{W}$ das gleiche asymptotische Verhalten wie $\mathfrak{S}_{\infty}$ und $\mathfrak{S}_{0}$ nach (12). Die Matrixelemente in (14) sind entsprechend (9) und (11)

$$
\begin{aligned}
{\left[\tilde{\mathfrak{V}}_{\infty}\right]_{i k} } & =\left(\tilde{\psi}_{i}^{*} V_{\infty} \tilde{\psi}_{k}\right), & {[\tilde{\mathfrak{M}}]_{i k} } & =\left(\tilde{\psi}_{i}^{*} \tilde{\psi}_{k}\right), \\
{\left[\tilde{\mathfrak{V}}_{0}\right]_{i k} } & =\left(\tilde{\Phi}_{i}^{*} V_{0} \tilde{\Phi}_{k}\right), & \delta_{i k} & =\left(\tilde{\Phi}_{i}^{*} \tilde{\Phi}_{k}\right) .
\end{aligned}
$$

Auf weitere Vereinfachungen von $\tilde{S}_{\infty}$ (vgl. ${ }^{1}$ ) wollen wir nicht eingehen, sondern jetzt dazu übergehen, die beiden Methoden, die in den Darstellungen $(10 \mathrm{~b}),(13)$ oder (14) vorliegen, zu vereinigen.

Zu diesem Zweck verbinden wir die Zerlegung des Hamilton-Operators mittels einer Funktion $p(R)$, die von den Kernabständen abhängt und den folgenden Bedingungen genügen soll.

$$
\lim _{R \rightarrow \infty} p(R)=\infty, \quad \lim _{R \rightarrow 0} p(R)=0 .
$$

Mit ihrer Hilfe schreiben wir für $\mathcal{H}$

$$
\begin{gathered}
\mathcal{H}=\frac{\left(H_{0}+V_{0}\right)+\left(H_{\infty}+V_{\infty}\right) p}{1+p}=\frac{H_{0}+H_{\infty} p}{1+p}+V \\
\text { mit } \quad V=\frac{V_{0}+V_{\infty} p}{1+p},
\end{gathered}
$$

wobei $V$ für $R \rightarrow \infty$ oder $\rightarrow 0$ verschwindet. (17) geht für $R \rightarrow \infty$ oder $\rightarrow 0$ in die Formen (2) oder (3) mit verschwindendem Störpotential $V_{\infty}$ bzw. $V_{0}$ über. Wir haben also

$$
\begin{array}{lll}
R \rightarrow \infty, & \mathcal{H} \rightarrow H_{\infty}+V_{\infty}, & V_{\infty} \rightarrow 0, \\
R \rightarrow 0, & \mathcal{H} \rightarrow H_{0}+V_{0}, & V_{0} \rightarrow 0 .
\end{array}
$$

Für die Molekülfunktion $\Omega$ machen wir nach (5) einen ähnlichen Ansatz und schreiben

$$
\begin{aligned}
\Omega_{a} & =\sum_{r} \gamma_{r a} \chi_{r} \\
\chi_{r} & =\frac{\Phi_{r}+\psi_{r} p}{1+p},
\end{aligned}
$$

mit

der ebenfalls für $R \rightarrow \infty$ oder $\rightarrow 0$ in die Darstellungen (5) übergeht. Der weitere Rechenweg läuft jetzt sehr ähnlich wie oben. Entsprechend (8) erhalten wir jetzt

$$
|\mathfrak{S}-\widetilde{\subseteq} E|=0 \text {, }
$$

wo

$$
[\mathfrak{S}]_{i k}=\left(\chi_{i}^{*} \mathcal{H} \chi_{k}\right) \text { und }[\subseteq]_{i k}=\left(\chi_{i}^{*} \chi_{k}\right) \text {. }
$$

Diese Matrizen lassen sich ebenfalls in üblicher Weise zerlegen, wobei neben den Elementen (11) noch vier neue auftreten, nämlich

$$
\begin{aligned}
{\left[\mathfrak{U}_{0}\right]_{i k} } & =\left(\psi_{i}{ }^{*} V_{0} \Phi_{k}\right), \quad[\mathfrak{N}]_{i k}=\left(\psi_{i}^{*} \Phi_{k}\right), \\
{\left[\bar{U}_{\infty}\right]_{i k} } & =\left(\Phi_{i}^{*} V_{\infty} \psi_{k}\right), \text { und }[\overline{\mathfrak{N}}]_{i k}=\left(\Phi_{i}^{*} \psi_{k}\right) .
\end{aligned}
$$

Mit diesen erhält man dann an Stelle von $(10 \mathrm{~b})$ und (13)

$$
\begin{aligned}
& (1+p)^{2} \mathfrak{S}_{2}=\mathfrak{S}_{0}+p \mathscr{S}_{M}+p^{2} \mathfrak{S}_{\infty}, \\
& (1+p)^{2} \widetilde{S}=\mathfrak{F}+p(\mathfrak{N}+\overline{\mathfrak{N}})+p^{2} \mathfrak{M} \text {, }
\end{aligned}
$$

wobei

$$
\begin{gathered}
\mathfrak{S}_{M}=\frac{1}{2}\left[\mathfrak{N}\left(\mathfrak{W}_{0}+\mathfrak{W}_{\infty}\right)+\left(\mathfrak{W}_{0}+\mathfrak{W}_{\infty}\right) \mathfrak{R}\right] \\
+\frac{1}{2}\left[\mathfrak{U}_{0}+\mathfrak{U}_{0}^{+}+\mathfrak{U}_{\infty}+\overline{\mathfrak{U}}_{\infty}^{+}\right] .
\end{gathered}
$$

Wegen der Hermizität von $\mathcal{H}$ kann für $\mathfrak{\mathcal { Y }}_{M}$ auch geschrieben werden

$$
\begin{aligned}
& \mathfrak{H}_{M}=\left[\mathfrak{N} \mathfrak{W}_{0}+\mathfrak{W}_{0} \mathfrak{N}\right]+\mathfrak{U}_{0}+\mathfrak{U}_{0}^{+}, \\
& \mathfrak{S}_{M}=\left[\mathfrak{N} \mathfrak{W}_{\infty}+\mathfrak{W}_{\infty} \mathfrak{N}\right]+\overline{\mathfrak{U}}_{\infty}+\overline{\mathfrak{U}}_{\infty}^{+} .
\end{aligned}
$$

Für $R \rightarrow \infty$ oder $\rightarrow 0$ geht (22) wieder in (10 a) oder $(10 \mathrm{~b})$ über.

Wir haben mit (22) also erreicht, daß die beiden Grenzfälle, die getrennten Atome und das vereinigte Atom, durch eine Gleichung beschrieben werden und alle weiteren Rechnungen relativer Elektronenenergien, bezogen auf die beiden Grenzfälle, durchgeführt werden können.

Ebenso wie in (14) führt die Verwendung von Näherungsfunktionen $\tilde{\chi}_{r}$ auf $\tilde{\mathfrak{S}}$ und $\tilde{\mathfrak{S}}$, wobei die Funktionen neben der zu erfüllenden Normierbarkeit sonst, ebenso wie $p(R)$, weitgehendst willkürlich sind.

Es ist somit zu erwarten, daß mit

$$
\begin{aligned}
& (1+p)^{2} \tilde{\mathfrak{Y}}=\tilde{\mathscr{S}}_{0}+p \tilde{\mathscr{Y}}_{M}+p^{2} \tilde{\mathfrak{S}}_{\infty}, \\
& (1+p)^{2} \tilde{\widetilde{S}}=\left(\mathfrak{E}+p(\tilde{\mathfrak{\Re}}+\tilde{\mathfrak{\Re}})+p^{2} \tilde{\mathfrak{M}}_{l}\right.
\end{aligned}
$$

eine im gesamten Bereich der Atomabstände ausreichende Näherung erreicht worden ist. $\tilde{\Phi}_{r}, \tilde{\psi}_{r}$ sowie $p(R)$ könnten im Prinzip noch Parameter enthalten, die durch Energievariation bestimmt werden, da die Form von $p$, außer den Forderungen (16), für $\mathcal{H}$ gleichgültig sind.

Es sei allerdings darauf hingewiesen, daß die Energievariation mit Hilfe von (19) kein Ritz-Verfahren in dem Sinne ist, daß die erhaltenen Energiewerte obere Grenzen für die wirklichen Eigenwerte sind. Durch die Beibehaltung von $\mathfrak{W}_{0}$ und $\mathfrak{W}_{\infty}$ in (10 a) und $(10 \mathrm{~b})$, neben den Näherungsfunktionen $(22 \mathrm{~b})$, 
kann es grundsätzlich möglich sein, daß die Energiewerte etwas tiefer als die wirklichen liegen.

$\mathrm{Da}$ dies bisher in der Form (8a) noch nicht erhalten worden ist ${ }^{3}$, liegt daran, daß die verwendeten Näherungsfunktionen im Bereich der vorkommenden Bindungsabstände noch so schlechte Näherungen sind, daß die Justierung der Energiekurven durch $\mathfrak{W}_{\infty}$ noch nicht ausreicht, diese unter der wirklichen verlaufen zu lassen. Auch aus diesem Grunde darf man erwarten, daß die Mitberücksichtigung von $\mathfrak{W}_{0}$ gute Ergebnisse liefert.

\section{Anhang}

Das Verfahren sei am einfachsten Beispiel des $\mathrm{H}_{2}{ }^{+}$. Moleküls erläutert. Hier ergibt sich für (2 a) und (3 a)

$$
\begin{aligned}
& H_{\infty}=-\frac{1}{2} \Delta-\frac{1}{r_{\mathrm{a}}} \text { bzw. }-\frac{1}{2} \Delta-\frac{1}{r_{\mathrm{b}}}, \\
& V_{\infty}=-\frac{1}{r_{\mathrm{b}}} \quad \text { bzw. }-\frac{1}{r_{\mathrm{a}}}, \\
& H_{0}=-\frac{1}{2} \Delta-\frac{2}{r_{\mathrm{c}}}, \\
& V_{0}=\frac{2}{r_{\mathrm{c}}}-\frac{1}{r_{\mathrm{a}}}-\frac{1}{r_{\mathrm{b}}} .
\end{aligned}
$$

Das vereinigte Atom ist ein $\mathrm{He}^{+}$an der Stelle $\mathrm{c}$ in der Mitte zwischen den Protonen a und b, deren Abstand $R$ beträgt.

Wir wollen den groben Ansatz

$$
\Omega=\frac{\Phi+p \psi}{1+p}
$$

verwenden und nur den Grundzustand bestimmen. Als Funktionen haben wir somit

$$
\begin{gathered}
\Phi=\sqrt{\frac{8}{\pi}} e^{-2 r_{\mathrm{c}}}, \quad \psi=\sqrt{\frac{1}{2 \pi(1+S)}}\left(e^{-r_{\mathrm{a}}}+e^{-r_{\mathrm{b}}}\right), \\
S=e^{-R}\left(1+R+\frac{1}{3} R^{2}\right),
\end{gathered}
$$

und es ist in atomaren Einheiten

$$
W_{\infty}=-0,5, \quad W_{0}=-2,0 .
$$

Damit ergibt sich für (10 a) und (10 b) bzw. (13)

$$
E_{0}=2,0-4 / R \cdot\left[1-(1+R) e^{-2 R}\right]
$$

und

$$
E_{\infty}=-0,5-\frac{1 / R \cdot\left[1-(1+R) e^{-2 R}\right]+(1+R) e^{-R}}{1+S} ;
$$

diese Funktionen sind in die Abb. 1 im Vergleich zur strengen Kurve $E$ eingezeichnet worden ${ }^{4}$.

3 R. Pauncz, Acta Phys. Hung. IV/3, 237 [1954].

4 E. Hylleraas, Z. Phys. 79, 739 [1931]; E. Teller, Z. Phys. 61, 458 [1930]; O. Burrau, Kgl. Danske Vidensk. Selsk. 7, 1 [1927].

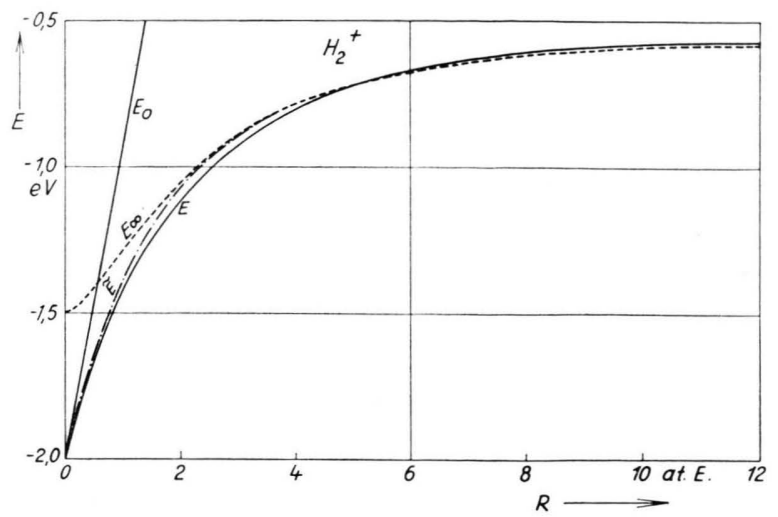

Abb. 1. Elektronenenergie des $\mathrm{H}_{2}{ }^{+}$-Moleküls als Funktion des Kernabstandes $R$.

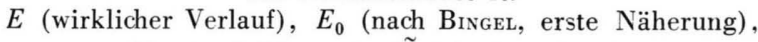
$E^{\infty}$ (nach Moffitт), $\tilde{E}$ (hier berechnet).

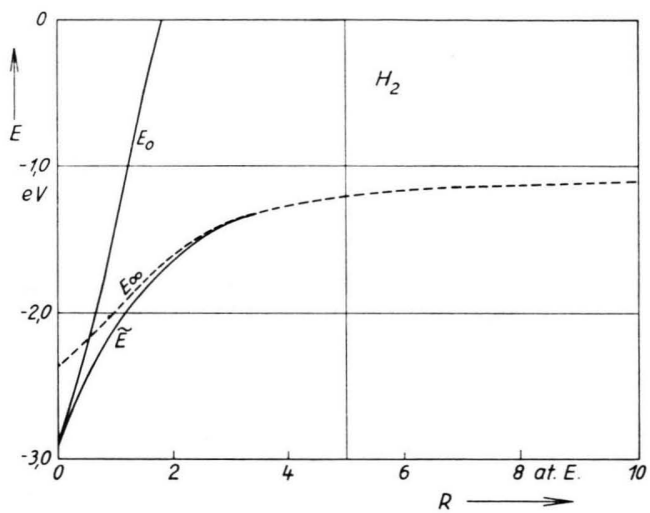

Abb. 2. Elektronenenergie des He-Moleküls als Funktion des Kernabstandes $R$.

$E_{0}$ (nach Bingel, erste Näherung), $E^{\infty}$ (nach Moffitt), $\tilde{E}$ (hier berechnet).

Nach (22 b) ergibt sich

$$
\tilde{E}=\frac{E_{0}+2 p E_{M}+p^{2} E_{\infty}}{1+2 M p+p^{2}}
$$

mit

$$
\begin{aligned}
M & =\frac{R^{3}}{2} \sqrt{\frac{1}{1+S}} f\left(\frac{R}{2}, R\right), \\
E_{M} & =-0,5 M-\frac{4}{\pi} \sqrt{\frac{1}{1+S}} \int \frac{1}{r_{\mathrm{b}}} e^{-2 r_{\mathrm{c}}-r_{\mathrm{a}}} \mathrm{d} \tau
\end{aligned}
$$

und $^{5}$

$R^{3} \pi f(\alpha, \beta)=\int e^{-\left(\alpha r_{\mathrm{a}}+\beta r_{\mathrm{b}}\right)} \mathrm{d} \tau$,

$R^{2} \pi g(\alpha, \beta)=\int \frac{1}{r_{\mathrm{a}}} e^{-\left(\alpha r_{\mathrm{a}}+\beta r_{\mathrm{b}}\right)} \mathrm{d} \tau$.

5 H. Preuss, „Integraltafeln zur Quantenchemie I“, SpringerVerlag 1956. 
Das Dreizentrenintegral wurde ausreichend abgeschätzt und $p$ variiert. Die damit erhaltenen $E$-Werte nach (26) sind ebenfalls in Abb. 1 graphisch dargestellt. Als Bindungsenergie ergibt sich etwa $-1,95 \mathrm{eV}$ bei einem Kernabstand von 2,3 at. E., während die entsprechenden Werte für $E_{\infty}-1,75 \mathrm{eV}$ und $R=2,6$ at. E. betragen. Für $E_{0}$ tritt noch keine Bindung auf. Die wirklichen Werte für $\mathrm{H}_{2}^{+}$sind $-2,78 \mathrm{eV}$ und 2,0 at. E.

Für $\mathrm{H}_{2}$ wurde der Ansatz (27) verwendet

$$
\Omega=\frac{\Phi+p \psi}{1+p}
$$

mit

$$
\begin{gathered}
\Phi=\frac{\lambda^{3}}{\pi} e^{-\lambda\left(r_{\mathrm{c} 1}+r_{\mathrm{c} 2}\right)}, \quad \psi=\frac{e^{-\left(r_{\mathrm{a} 1}+r_{\mathrm{b} 2}\right)+e^{-\left(r_{\mathrm{a} 2}+r_{\mathrm{b} 1}\right)}}}{\pi \sqrt{2\left(1+S^{2}\right)}}, \\
\lambda=1,70 .
\end{gathered}
$$

Der Hamilton-Operator wurde in folgender Weise zerlegt

$$
\begin{aligned}
& H_{\infty}=-\frac{1}{2} \Delta_{1}-\frac{1}{2} \Delta_{2}-\frac{1}{r_{\mathrm{a} 1}}-\frac{1}{r_{\mathrm{b} 2}} \\
& \text { bzw. }-\frac{1}{2} \Delta_{1}-\frac{1}{2} \Delta_{2}-\frac{1}{r_{\mathrm{a} 2}}-\frac{1}{r_{\mathrm{b} 1}}, \\
& V_{\infty}=-\frac{1}{r_{\mathrm{a} 2}}-\frac{1}{r_{\mathrm{b} 1}}+\frac{1}{r_{12}} \text { bzw. }-\frac{1}{r_{\mathrm{a} 1}}-\frac{1}{r_{\mathrm{b} 2}}+\frac{1}{r_{12}}, \\
& H_{0}=-\frac{1}{2} \Delta_{1}-\frac{1}{2} \Delta_{2}-\frac{2}{r_{\mathrm{c} 1}}-\frac{2}{r_{\mathrm{c} 2}}+\frac{1}{r_{12}}, \\
& V_{0}=\frac{2}{r_{\mathrm{c} 1}}-\frac{1}{r_{\mathrm{a} 1}}-\frac{1}{r_{\mathrm{b} 1}}+\frac{2}{r_{\mathrm{c} 2}}-\frac{1}{r_{\mathrm{a} 2}}-\frac{1}{r_{\mathrm{b} 2}} .
\end{aligned}
$$

Damit ergab sich dann (s. Abb. 2)

$$
\tilde{E}=\frac{E_{0}+2 p E_{M}+p^{2} E_{\infty}}{1+2 M p+p^{2}}
$$

mit

$$
\begin{aligned}
& E_{\infty}=-1,0+\frac{C+A}{1+S^{2}}, \\
& E_{0}=-2,903+4 \lambda\left\{1-\frac{g(0, R \lambda)}{g(2 \lambda R, 0)}\right\}, \\
& E_{M}=-2,903 M+\sqrt{\frac{2}{1+S^{2}} \lambda^{3}\left(\frac{R}{2}\right)^{3} f\left(\frac{R \lambda}{2}, \frac{R}{2}\right)} \\
& \left.\cdot \cdot \frac{R^{2}}{2} g\left(\frac{R \lambda}{2}, \frac{R}{2}\right)-G\right\}, \\
& G=\frac{R^{5}}{32} f\left(\frac{R \lambda}{2}, \frac{R}{2}\right)\left\{\lambda^{3} g(0, R \lambda)+4 g(0,2 R)\right\},
\end{aligned}
$$

wobei

$$
\begin{aligned}
& C=-\left\{R^{2}[g(2 R, 2 R)+g(0,2 R)]+R^{3} f(2 R, 2 R)\right\}, \\
& A=R^{5}[a(R, R, R, R)-2 g(R, R) f(R, R)],
\end{aligned}
$$$$
a(R, R, R, R)=\int e^{-\left(r_{\mathrm{a} 1}+r_{\mathrm{b} 1}+r_{\mathrm{a} 2}+r_{\mathrm{b} 2}\right) r_{12}} \mathrm{~d} \tau_{1} \mathrm{~d} \tau_{2},
$$

und

$$
M=\sqrt{\frac{2}{1+S^{2}}} \lambda^{3}(R / 2)^{6}[f(R \lambda / 2, R / 2)]^{2}
$$

$$
\begin{array}{ll}
W_{0}=-2,903 & \text { (Heliumenergie }) \\
W_{\infty}=-1,000 & (2 \times \text { Wasserstoffenergie })
\end{array}
$$

bedeuten.

Nach Variation von $p$ erhält man im Minimum der Bindungsenergie $-4,71 \mathrm{eV}$ bei einem Kernabstand von $R=1,466$ at. E. $E_{\infty}$ allein liefert nur $-4,35 \mathrm{eV}$ und 1,40 at. E. ${ }^{3}$. Die wirklichen Werte betragen $-4,72 \mathrm{eV}$ und 1,40 at. E.

Frau I. Funke danke ich herzlich für die hierzu durchgeführten Rechnungen.

\title{
Zur Komplanarität aromatischer Verbindungen*
}

\author{
Von H. Preuss \\ Aus dem Max-Planck-Institut für Physik, Göttingen \\ (Z. Naturforschg. 12 a, 603-608 [1957] ; eingegangen am 6. Juni 1957)
}

\begin{abstract}
Mit Hilfe des sogenannten Näherungsverfahrens werden in Näherung die Winkelabhängigkeiten der Torsionsenergien von $\pi$-Elektronensystemen bei der offenen Kohlenstoffkette, beim Triphenylmethyl und beim Diphenylmethyl abgeschätzt. Während sich in dieser Form der Näherung bei der Kohlenstoffkette eine reine Cosinus-Abhängigkeit ergeben kann, ist der Zusammenhang zwischen Resonanzenergie und Torsionswinkel bei den Radikalen komplizierter und zeigt, daß eine Verdrehung der Phenyle bis etwa $40^{\circ}$ die Resonanzenergie um rund $32 \%$, beim Triphenylmethyl um etwa $30 \%$ vermindert.
\end{abstract}

Eine wichtige Frage in der Quantentheorie der chemischen Bindung ist die nach den bei Torsionen von Molekülen auftretenden Energien und deren Abhängigkeiten vom Drehwinkel ${ }^{1}$. Bekanntlich benutzt man in diesem Zusammenhang in der organi-

* Vorgetragen bei der 56. Tagung der Bunsengesellschaft, Kiel 1957. schen Chemie den Begriff der freien Drehbarkeit, wobei dieser dann anzuwenden ist, wenn sich die Hälften eines Moleküls um die Valenzrichtung, welche sie verbindet, ohne Energieänderung gegeneinander verdrehen können. In Wirklichkeit liegt aller-

1 Als Erster hat E. Hückel, Z. Phys. 60, 423 [1930], die Frage theoretisch behandelt. 